\title{
INTERPOLATING FUNCTIONS ASSOCIATED WITH SECOND-ORDER DIFFERENTIAL EQUATIONS
}

\author{
WILLIAM F. TRENCH
}

\begin{abstract}
Functions are exhibited which interpolate the magnitude of a solution $y$ of a linear, homogeneous, second-order differential equation at its critical points, $\left|y^{\prime}\right|$ at the zeros of $y$, and $\left|\int_{x_{0}}^{x} y(t) h(t) d t\right|$ at the zeros of $y$. Except for a normalization condition, the interpolating functions are independent of the specific solution $y$. A theorem similar in its conclusions to the Sonin-Pólya-Butlewski theorem is presented and examples are given.
\end{abstract}

1. Introduction. Suppose $y_{1}$ and $y_{2}$ form a fundamental system for the differential equation

$$
\left(p(x) y^{\prime}\right)^{\prime}+q(x) y=0, \quad a<x<b,
$$

where we assume throughout that $p$ and $q$ are continuous and $p>0$ on $(a, b)$. Let $y=A y_{1}+B y_{2}$ be an arbitrary nontrivial solution of (1), normalized so that $A^{2}+B^{2}=1$. We will exhibit a function, independent of $A$ and $B$, which interpolates $|y|$ at the critical points of $y$, and others, also independent of $A$ and $B$, which interpolate $\left|y^{\prime}\right|$ and $\left|\int_{x_{0}}^{x} y(t) h(t) d t\right|$ at the zeros of $y$.

\section{Preliminary lemmas.}

Lemma 1. Suppose $f_{1}, f_{2}, g_{1}$ and $g_{2}$ are real numbers, with $f_{1}^{2}+f_{2}^{2}>0$. Let

$$
f=A f_{1}+B f_{2}, \quad g=A g_{1}+B g_{2},
$$

with

$$
A^{2}+B^{2}=1
$$

Then

$$
|g|=\left|f_{1} g_{2}-f_{2} g_{1}\right| /\left(f_{1}^{2}+f_{2}^{2}\right)^{1 / 2} \text { if } f=0 .
$$

Proof. The conclusion follows from the identity

$$
\left(A^{2}+B^{2}\right)\left(f_{1} g_{2}-f_{2} g_{1}\right)^{2}=\left(f g_{2}-f_{2} g\right)^{2}+\left(f_{1} g-f g_{1}\right)^{2}
$$

which can be derived by solving (2) for $A$ and $B$, squaring and adding, and then multiplying both sides of the resulting equation by $\left(f_{1} g_{2}-f_{2} g_{1}\right)^{2}$. Although this seemingly requires the assumption that $f_{1} g_{2}-f_{2} g_{1} \neq 0$, it is easily verified that (4) holds for all values of the quantities appearing in it. Invoking (3) yields (5).

In the next two lemmas $y_{1}$ and $y_{2}$ need not be solutions of (1).

Received by the editors July 2, 1978 and, in revised form, January 22, 1979.

AMS (MOS) subject classifications (1970). Primary 34C10.

Key words and phrases. Zeros, interpolation, cylinder function, Sonin-Pólya-Butlewski theorem. 
LEMMA 2. Let $y_{1}$ and $y_{2}$ be differentiable and suppose their Wronskian, $W=y_{1} y_{2}^{\prime}-y_{2} y_{1}^{\prime}$, has no zeros in $(a, b)$. Let

$$
y=A y_{1}+B y_{2} \quad\left(A^{2}+B^{2}=1\right) \text {. }
$$

Then

$$
|y(\bar{x})|=|W(\bar{x})| /\left[\left(y_{1}^{\prime}(\bar{x})\right)^{2}+\left(y_{2}^{\prime}(\bar{x})\right)^{2}\right]^{1 / 2} \text { if } \bar{x} \in(a, b) \text { and } y^{\prime}(\bar{x})=0,
$$

and

$$
\left|y^{\prime}(\bar{x})\right|=|W(\bar{x})| /\left[y_{1}^{2}(\bar{x})+y_{2}^{2}(\bar{x})\right]^{1 / 2} \text { if } \bar{x} \in(a, b) \text { and } y(\bar{x})=0 .
$$

Proof. To obtain (7), take $f_{i}=y_{i}^{\prime}(\bar{x})$ and $g_{i}=y_{i}(\bar{x})(i=1,2)$ in Lemma 1 . To obtain (8), take $f_{i}=y_{i}(\bar{x})$ and $g_{i}=y_{i}^{\prime}(\bar{x})$ in Lemma 1 . The nonvanishing of $W$ guarantees that the denominators in (7) and (8) are nonzero.

LEMMA 3. Suppose $y_{1} h$ and $y_{2} h$ are locally integrable and $y_{1}^{2}+y_{2}^{2}>0$ on $(a, b)$. Define

$$
s(x)=\int_{x_{0}}^{x}\left[y_{1}(t) y_{2}(x)-y_{2}(t) y_{1}(x)\right] h(t) d t, \quad a<x_{0}, x<b,
$$

and let $y$ satisfy (6). Then

$$
\left|\int_{x_{0}}^{\bar{x}} y(t) h(t) d t\right|=\frac{|s(\bar{x})|}{\left[y_{1}^{2}(\bar{x})+y_{2}^{2}(\bar{x})\right]^{1 / 2}} \quad \text { if } \bar{x} \in(a, b) \text { and } y(\bar{x})=0 \text {. }
$$

The conclusion also holds with $x_{0}=a$ if $\int_{a} y_{i}(t) h(t) d t$ exists $(i=1,2)$, or with $x_{0}=b$ if $\int^{b} y_{i}(t) h(t) d t$ exists $(i=1,2)$.

Proof. Take $f_{i}=y_{i}(\bar{x})$ and $g_{i}=\int_{x_{0}}^{\bar{x}} y_{i}(t) h(t) d t(i=1,2)$ in Lemma 1.

3. Main results. If $y_{1}$ and $y_{2}$ are linearly independent solutions of (1), then

$$
y_{1} y_{2}^{\prime}-y_{2} y_{1}^{\prime}=k / p \quad(k=\text { constant } \neq 0) \text {. }
$$

This and Lemma 2 yield the following theorem.

THEOREM 1. Let $y_{1}$ and $y_{2}$ be solutions of (1) satisfying (11), and suppose $y$ satisfies (6). Then the function

$$
I_{1}=|k| / p\left[\left(y_{1}^{\prime}\right)^{2}+\left(y_{2}^{\prime}\right)^{2}\right]^{1 / 2}
$$

interpolates $|y|$ at the critical points of $y$, and the function

$$
I_{2}=|k| / p\left(y_{1}^{2}+y_{2}^{2}\right)^{1 / 2}
$$

interpolates $\left|y^{\prime}\right|$ at the zeros of $y$.

This theorem can essentially be obtained by applying known transformations to (1), although the argument presented above is simpler and appears to require fewer assumptions. The substitutions of

$$
t=\int^{x} \frac{d u}{p(u)}, \quad Y(t)=y(x)
$$


transform (1) into

$$
d^{2} Y / d t^{2}+Q(t) Y=0
$$

where $Q(t)=p(x) q(x)$. If $y_{1}$ and $y_{2}$ are as defined at the beginning of this section and $Y_{i}(t)=y_{i}(x)(i=1,2)$, let $f(t)=Y_{1}^{2}(t)+Y_{2}^{2}(t)$. L. Lorch and P. Szegö [3] have shown that the substitutions

$$
z=\int^{t} \frac{d v}{f(v)}, \quad u(z)=(f(t))^{1 / 2} Y(t)
$$

transform (14) into $d^{2} u / d z^{2}+k^{2} u=0$. Therefore, the general solution of (1) is

$$
y(x)=C\left[y_{1}^{2}(x)+y_{2}^{2}(x)\right]^{1 / 2} \sin \left(k \int^{t} \frac{d u}{f(u)}\right),
$$

with $t$ related to $x$ as in (13). (This formula was given by Boruivka [1, p. 43] for the case where $p(x)=1$.) By differentiating (15) while recalling (13), and noting that $y(x)=0$ if and only if $\sin \left(k \int^{t} d u / f(u)\right)=0$ (and so $\cos \left(k \int^{t}\right.$ $d u / f(u))= \pm 1$ ), it can be shown that a constant multiple of $I_{2}$ interpolates $\left|y^{\prime}\right|$ at the zeros of $y$.

Formally, the equation $\left(u^{\prime} / q\right)^{\prime}+u / p=0$ has solutions $u=p y^{\prime}$, where $y$ satisfies (1). Applying the procedure of the last paragraph to this equation leads to a general formula for $y^{\prime}$ (also given by Boruiva for the case where $p(x)=1[1, \mathrm{p} .43])$, from which it can be shown that a constant multiple of $I_{1}$ interpolates $|y|$ at the zeros of $y^{\prime}$. It would appear that this derivation of $I_{1}$ requires the additional assumption that $p$ has no zeros on $(a, b)$.

It should be observed that the use of the function $y_{1}^{2}+y_{2}^{2}$ for obtaining qualitative properties of solutions of (1) occurs in many places in the literature; for examples, see [2, pp. 515-519], [4], [5] and [6].

THEOREM 2. Suppose (1) has linearly independent solutions $y_{1}$ and $y_{2}$ such that the function $F=q\left(y_{1}^{2}+y_{2}^{2}\right)^{\prime}$ does not change sign on $(a, b)$. Let $\left\{x_{n}\right\}$ be an increasing sequence of critical points of an arbitrary nontrivial solution $y$ of (1). Then the sequence $\left\{\left|y\left(x_{n}\right)\right|\right\}$ is nondecreasing if $F>0$, or nonincreasing if $F<0$. The monotonicity of $\left\{\left|y\left(x_{n}\right)\right|\right\}$ is strict if $F$ is not identically zero on any subinterval of $(a, b)$.

Proof. Assume without loss of generality that $y$ is normalized as in (6); then Theorem 1 implies that $\left|y\left(x_{n}\right)\right|=I_{1}\left(x_{n}\right)$, with $I_{1}$ as in (12). Differentiating $I_{1}$ and noting that $y_{1}$ and $y_{2}$ satisfy (1) yields $I_{1}^{\prime}=p F I_{1}^{3} / 2 k^{2}$. This implies the conclusion.

THEOREM 3. Suppose $y_{1}$ and $y_{2}$ are solutions of (1) which satisfy (11), that $a<x_{0}<b$, and that $s$ satisfies

$$
\left(p(x) s^{\prime}\right)^{\prime}+q(x) s=k h(x), \quad s\left(x_{0}\right)=s^{\prime}\left(x_{0}\right)=0,
$$

where $h$ is continuous on $(a, b)$. Then (10) holds, with $y$ as in (6). This is also true with $x_{0}=a$ if $\int_{a} y_{i}(t) h(t) d t$ exists $(i=1,2)$, or with $x_{0}=b$ if $\int^{b} y_{i}(t) h(t) d t$ exists $(i=1,2)$. 
Proof. By variation of parameters, $s$ is as in (9), so Lemma 3 implies the conclusion.

4. Examples. Let (1) be Bessel's equation,

$$
\left(x y^{\prime}\right)^{\prime}+\frac{1}{x}\left(x^{2}-\nu^{2}\right) y=0
$$

with $y_{1}=J_{\nu}$ and $y_{2}=Y_{\nu}$, the Bessel functions of the first and second kinds, and let

$$
\bigodot_{\nu}=A J_{\nu}+B Y_{\nu} \quad\left(A^{2}+B^{2}=1\right)
$$

be an arbitrary normalized cylinder function.

EXAMPLE 1. Since the Wronskian of $y_{1}$ and $y_{2}$ in this case is

$$
W(x)=J_{\nu}(x) Y_{\nu}^{\prime}(x)-Y_{\nu}(x) J_{\nu}^{\prime}(x)=2 / \pi x
$$

$[8$, p. 76], Theorem 1 implies that

$$
\left|\bigodot_{\nu}(\bar{x})\right|=\frac{2}{\pi \bar{x}\left[\left(J_{\nu}^{\prime}(\bar{x})\right)^{2}+\left(Y_{\nu}^{\prime}(\bar{x})\right)^{2}\right]^{1 / 2}} \text { if } \bar{x}>0 \text { and } \bigodot_{\nu}^{\prime}(\bar{x})=0
$$

and

$$
\left|\varrho_{\nu}^{\prime}(\bar{x})\right|=\frac{2}{\pi \bar{x}\left[\left(J_{\nu}(\bar{x})\right)^{2}+\left(Y_{\nu}(\bar{x})\right)^{2}\right]^{1 / 2}} \text { if } \bar{x}>0 \text { and } \bigodot_{\nu}(\bar{x})=0 .
$$

It is perhaps worth noting that both interpolating functions are rational if $\nu=k+1 / 2$, where $k$ is an integer [8, p. 297]. Since $x\left(J_{v}^{2}+Y_{v}^{2}\right)$ increases if $\nu>1 / 2$ and decreases if $\nu<1 / 2$ [8, p. 466], (18) implies that if $\left\{x_{n}\right\}$ is an increasing sequence of positive zeros of $\mathcal{C}_{p}$, then the sequence $\left\{\left|\mathcal{C}_{p}^{\prime}\left(x_{n}\right)\right|\right\}$ decreases if $\nu>1 / 2$ and increases if $\nu<1 / 2$. Since $J_{\nu}^{2}+Y_{\nu}^{2}$ decreases for all $\nu$ [8, p. 466], (18) also implies that the sequence $\left\{x_{n}\left|\mathcal{C}_{\nu}^{\prime}\left(x_{n}\right)\right|\right\}$ increases for all $\nu$.

EXAMPLE 2. If $\bigodot_{\nu}$ is a cylinder function as in (16), then $\int_{0}^{x} t^{\mu} C_{\nu}(t) d t$ exists if $\mu \pm \nu>-1$. From (11) and (17), the initial value problem associated with this integral according to Theorem 3 is

$$
\left(x s^{\prime}\right)^{\prime}+\frac{1}{x}\left(x^{2}-\nu^{2}\right) s=2 x^{\mu} / \pi, \quad s(0)=s^{\prime}(0)=0,
$$

which has the solution $s=2 s_{\mu v} / \pi$, where $s_{\mu \nu}$ is Lommel's function of the first kind [8, pp. 345-346]. Now (10) implies that

$$
\left|\int_{0}^{x} t^{\mu} C_{\nu}(t) d t\right|=\frac{2\left|s_{\mu \nu}(\bar{x})\right| / \pi}{\left[J_{\nu}^{2}(\bar{x})+Y_{\nu}^{2}(\bar{x})\right]^{1 / 2}} \text { if } \bar{x}>0 \text { and } C_{\nu}(\bar{x})=0 .
$$

Example 3. If $\mu<1 / 2$ and $C_{\nu}$ is as in (16), then $\int_{x}^{\infty} t^{\mu} C_{\nu}(t) d t$ exists for $x>0$. The initial value problem associated with this integral according to Theorem 3 is

$$
\left(x s^{\prime}\right)^{\prime}+\frac{1}{x}\left(x^{2}-\nu^{2}\right) s=2 x^{\mu} / \pi, \quad s(\infty)=s^{\prime}(\infty)=0
$$

which has the solution $s=2 S_{\mu \nu} / \pi$, where $S_{\mu \nu}$ is Lommel's function of the 
second kind [8, p. 347]. Therefore, Theorem 3 implies that

$$
\left|\int_{\bar{x}}^{\infty} t^{\mu} \bigodot_{\nu}(t) d t\right|=\frac{2\left|S_{\mu \nu}(\bar{x})\right| / \pi}{\left[J_{\nu}^{2}(\bar{x})+Y_{\nu}^{2}(\bar{x})\right]^{1 / 2}} \quad \text { if } \bar{x}>0 \text { and } \bigodot_{\nu}(\bar{x})=0 .
$$

EXAMPLE 4. Theorem 2 and the fact that $J_{\nu}^{2}+Y_{\nu}^{2}$ decreases imply that the successive maxima of $\left|e_{\nu}\right|$ on $(\nu, \infty)$ form a decreasing sequence. This is a known result $[8$, p. 488].

The conclusion of Theorem 2 is similar to that of the Sonin-Pólya-Butlewski theorem [7, p. 166], which says that if $p>0, q>0, p$ and $q$ are continuously differentiable on $(a, b)$, and $p q$ is monotonic, then $\left\{\left|y\left(x_{n}\right)\right|\right\}$ is monotonic in the opposite sense. By considering transformed versions of Bessel's equation and using known monotonicity properties of $x\left(J_{\nu}^{2}+Y_{\nu}^{2}\right)$ and $\left(x^{2}-v^{2}\right)^{1 / 2}\left(J_{v}^{2}+Y_{v}^{2}\right)$ [8, p. 446], it is possible to obtain from Theorem 2 monotonicity properties of sequences of maxima of $x^{1 / 2}\left|\mathcal{C}_{\nu}\right|$ and $\left(x^{2}-\right.$ $\left.\nu^{2}\right)^{1 / 4}\left|\mathcal{C}_{\nu}\right|$; however, the differential equations in question also satisfy the hypotheses of the Sonin-Pólya-Butlewski theorem, and the results are not new. The following contrived example presents a result implied by Theorem 2 that cannot be obtained from the Sonin-Pólya-Butlewski theorem.

EXAMPLE 5. If $g$ is positive and continuously differentiable on $(0, \infty)$, then

$$
y_{1}(x)=x \cos \left(\int_{0}^{x} g(t) d t\right) \text { and } y_{2}(x)=x \sin \left(\int_{0}^{x} g(t) d t\right)
$$

form a fundamental system for

$$
\left(\frac{y^{\prime}}{x^{2} g}\right)^{\prime}+\left(\frac{2}{x^{3} g}+\frac{g^{\prime}}{x^{2} g^{2}}+\frac{g}{x}\right) y=0, \quad x>0 .
$$

Clearly (19) is oscillatory if $\int^{\infty} g(t) d t=\infty$, and since $y_{1}^{2}(x)+y_{2}^{2}(x)=x^{2}$ is increasing, it satisfies the hypotheses of Theorem 2 on $(0, \infty)$ if $g^{\prime}>0$; therefore, if these assumptions hold and $\phi$ is any constant, then the absolute values of

$$
y(x)=x \cos \left(\phi+\int_{0}^{x} g(t) d t\right)
$$

at any increasing sequence of critical points of $y$ form an increasing sequence. The Sonin-Pólya-Butlewski theorem does not imply this, since

$$
p q=\frac{1}{x^{2} g}\left(\frac{2}{x^{3} g}+\frac{g^{\prime}}{x^{2} g^{2}}+\frac{g}{x}\right)
$$

need not be monotonic; for example, let

$$
g(x)=\int_{0}^{x}\left(2-\cos e^{t}\right) d t .
$$

5. Acknowledgment. The three paragraphs following Theorem 1 are due to the referee who kindly brought the matters discussed there to my attention. 


\section{REFERENCES}

1. O. Boruvka, Linear differential transformations of the second order, English Univ. Press, 1971 (German original, Lineare Differentialtransformationen 2. Ordnung, VEB, Berlin, 1967).

2. P. Hartman, Ordinary differential equations, 2nd ed., Hartman, Baltimore, Md., 1973.

3. L. Lorch and P. Szegö, Higher monotonicity properties of certain Sturm-Liouville functions, Acta Math. 109 (1963), 55-73.

4. L. Lorch, M. E. Muldoon and P. Szegö, Higher monotonicity properties of certain Sturm-Liouville functions. III, Canad. J. Math. 22 (1970), 1238-1265.

5. L. Lorch, M. E. Muldoon and P. Szegö, Higher monotonicity properties of certain Sturm-Liouville functions. IV, Canad. J. Math. 24 (1972), 349-368.

6. H. Milloux, Sur l'équation différentielle $x^{\prime \prime}+x A(t)=0$, Prace Mat.-Fiz. 41 (1933), 39-54.

7. G. Szegö, Orthogonal polynomials, 4th ed., Amer. Math. Soc. Colloq. Publ., vol. 23, Amer. Math. Soc., Providence, R. I., 1975.

8. G. N. Watson, A treatise on the theory of Bessel functions, 2nd ed., Cambridge Univ. Press, Cambridge, 1944.

Department of Mathematics, Drexel University, Philadelphia, Pennsylvania 19104 